\title{
Phagocytosis and killing ability of Candida albicans by blood leucocytes of healthy term and preterm babies
}

\author{
M. XANTHOU, E. VALASSI-ADAM, E. KINTZONIDOU, and N. MATSANIOTIS \\ From the Department of Paediatrics, University of Athens
}

\begin{abstract}
Xanthou, M., Valassi-Adam, E., Kintzonidou, E., and Matsaniotis, N. (1975). Archives of Disease in Childhood, 50, 72. Phagocytosis and killing ability of Candida albicans by blood leucocytes of healthy term and preterm babies. The phagocytosis and killing ability of leucocytes of 24 term and 22 preterm babies against Candida albicans were studied during the first 20 days of life because of the increased incidence of monilia infection at this time. The leucocytes of 14 adults aged 20 to 30 years served as controls. The phagocytosis ability of the leucocytes in adults, term, and preterm babies was not significantly different, mean values being respectively $66 \cdot 7 \%, 57 \%$, and $56 \cdot 9 \%$.

The killing ability of the leucocytes in term and preterm babies was lower when compared with that of adult leucocytes $(P<0.001$ for term and $P<0.01$ for preterm infants). The mean value in adults was $27.5 \%$, in term infants $9.7 \%$, and in preterm infants $9 \cdot 5 \%$.

It is suggested that as the addition of adult serum did not improve the candidacidal ability of newborn leucocytes, the killing defect should be sought in the leucocyte itself and not in serum factors.
\end{abstract}

Various functions of newborn leucocytes have been the subject of much recent work in order to explain the notorious susceptibility of neonates to infection. Particular attention has been paid to the ability of leucocytes to phagocytize and subsequently kill pathogenic micro-organisms like Esch. coli, Staphylococcus aureus, Pseudomonas aeruginosa, etc. Both these functions were found to be deficient and more so in the preterm infants mainly because of opsonic deficiencies in their serum. Addition of adult serum would in most instances correct the phagocytosis and killing defects (Forman and Stiehm, 1969; Miyamoto, 1965a, b; Dossett, Williams, and Quie, 1969; Park, Holmes, and Good, 1970; McCracken and Eichenwald, 1971).

The high incidence of monilia infection in the newborn has stimulated us to test these leucocyte functions with $C$. albicans; moreover, candida has a particular immunological interest as candidiasis often accompanies either a fall in host resistance or immunological defects.

Received 20 June 1974.

\section{Patients and methods}

The phagocytosis and candidacidal ability of leucocytes were studied in 24 term and 22 preterm babies. 14 adults 20 to 30 years of age were used as controls. Out of the 46 newborns, 4 term and 2 preterm babies were small-for-dates. 11 preterm babies had a gestational age $<32$ weeks, and $11>32$ weeks. The postnatal age ranged from 1 to 20 days with a mean of 6 days.

All babies were healthy at the time of the test apart from 11 term and 4 preterm babies who had jaundice but were otherwise doing very well. The aetiology of jaundice was $R$ incompatibility in 3, G6PD deficiency in 3, ABO incompatibility in 1 , and unknown origin in the remaining 8. Finally, all babies included in this study received prophylactic antibiotic therapy (penicillin and gentamicin for 7-10 days) because of prolonged rupture of membranes or because of poor means of transportation to our unit. The blood specimens were obtained either by venepuncture or from a free-flowing heel prick.

Phagocytosis and killing abilities were evaluated by the method described by Lehrer and Cline (1969) with minor modifications. Similar numbers of polymorphonuclear neutrophils and viable candidae in a mixture of Hanks's 
salt solution and patient's serum were incubated for 30 minutes at $37{ }^{\circ} \mathrm{C}$ and the percentage of intracellular candidae was determined for estimation of phagocytosis. The determination of killing ability was based on differential staining of nonviable candidae which take methylene blue dye and acquire a deep blue colour being thus clearly distinguished from viable organisms which do not take the stain and remain white. The method was chosen as more reliable and simpler than the ones relying on the counting of colonies on cultures.

Lehrer and Cline (1969) used $10 \mathrm{ml}$ of blood and suspended the cells in fetal calf serum and dextran. We had to minimize the amount of blood taken in newborn babies and worked with a maximum of $4 \mathrm{ml}$ from which we managed to separate an adequate number of leucocytes using dextran only. However, the test with smaller numbers of leucocytes and consequently candidae was more time consuming.

Phagocytosis was expressed as the number of phagocytosed candidae per 100 organisms. Candidacidal ability was expressed (a) as the number of nonviable candidae per 100 phagocytosed organisms, (b) as the number of nonviable candidae per 100 organisms (crude phagocytosis). No distinction was made regarding the type of phagocytosing leucocytes whether polymorphonuclear neutrophils, eosinophils, or monocytes.

Finally $\mathrm{C} 3$ levels were measured by radial immunodiffusion using $\beta_{1} \mathrm{C} / \beta_{1} \mathrm{~A}$ antiserum and home-made immunoplates.

\section{Results}

Phagocytosis and candidacidal ability of leucocytes were very similar in jaundiced, small-fordates, and healthy newborns. Therefore, all these babies were included in the same groups.

Phagocytosis. Adult leucocytes in their own serum had a mean phagocytosis value of $66 \cdot 7 \%$. Term and preterm babies' leucocytes in their own serum had a mean phagocytosis value of $57 \%$ and $56.9 \%$, respectively. The values in adults ranged from $41 \%$ to $94 \%$. An even larger range (23.6\%$92.5 \%$ ) was found in newborns (Fig. 1).

Although adult leucocytes tend to have a better ability to phagocytize $C$. albicans, no significant difference was found between their ability and that of term or preterm babies. In preterm babies no difference in phagocytosis was found between those of less than 32 weeks' gestation and those above it. The addition of adult serum did not affect to any appreciable degree the phagocytosis in term or preterm babies.

Candidacidal ability. The ability of adult leucocytes in their own serum to kill ingested $C$. albicans had a mean geometric value of $27.5 \%$. The newborn leucocytes in their own serum had a much lower candidacidal ability, the mean geometric

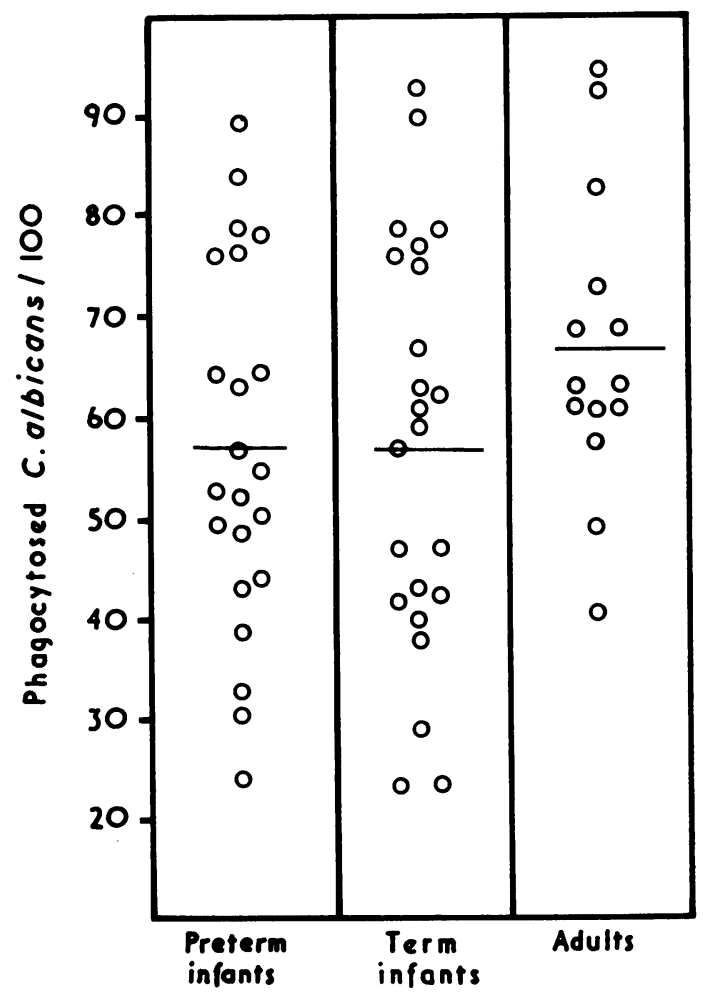

Fig. 1.-Percentage of Candida albicans cells phagocytosed in 30 minutes by leucocytes of adults, term, and preterm babies.

value for the term infants being $9 \cdot 7 \%$, and for the preterms $9.5 \%$. There was no difference in the candidacidal ability between term and preterm babies nor between preterm babies above and below 32 weeks of gestation. However, the difference in the candidacidal ability between adults and term babies as well as between adults and preterm babies was found to be highly significant $(P<0.001$ and $P<0.01$, respectively). The range of the killing ability of leucocytes was again wider in the newborns, some of whom had no candidacidal ability at all (Fig. 2). The addition of adult serum did not improve the candidacidal ability of the leucocytes in term or preterm babies.

Finally, regarding the crude candidacidal ability, the adults' leucocytes were found again to be significantly better when compared with that of the newborns.

Complement. C3 levels ranged from 22 $\mathrm{mg} / 100 \mathrm{ml}$ to $265 \mathrm{mg} / 100 \mathrm{ml}$ with a mean value of $99.6 \mathrm{mg} / 100 \mathrm{ml}$ in newborns. Though the $\mathrm{C} 3$ 


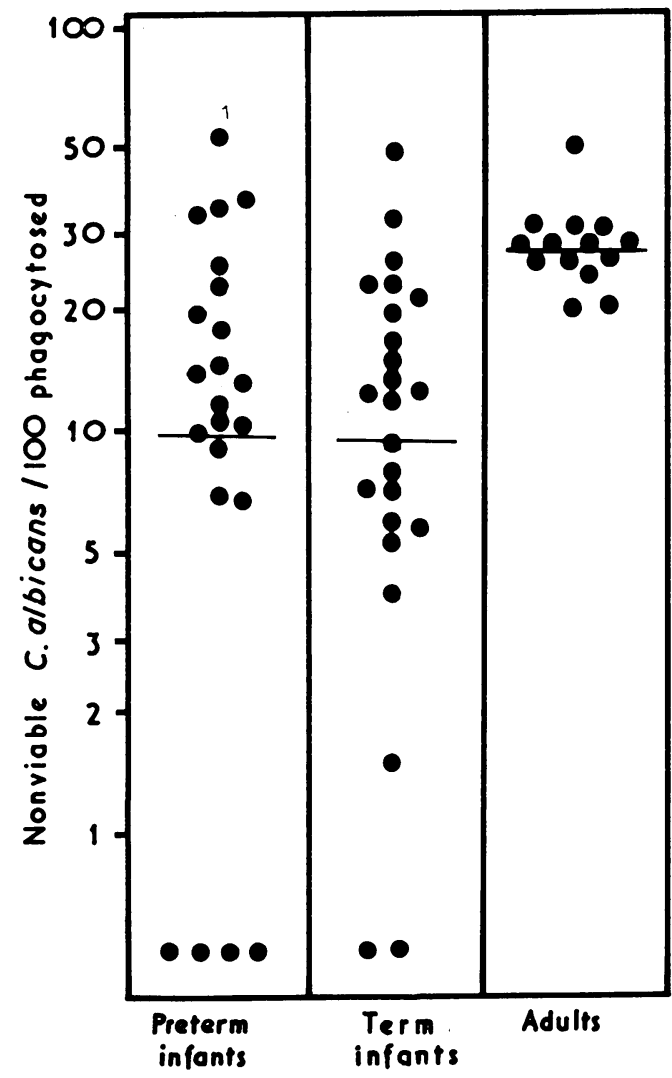

FIG. 2.-Percentage of Candida albicans cells killed in 1 hour by leucocytes of adults, term, and preterm babies plotted on a logarithmic scale.

component of complement tended to increase with gestational age, no significant statistical differences were found between term and preterm babies. The concentration of $\mathrm{C} 3$ did not show any correlation with phagocytosis or killing ability of newborn leucocytes.

Finally, the postnatal age did not have any correlation with the above functions of leucocytes.

\section{Discussion}

We have found that the phagocytosis of $C$. albicans by adult leucocytes, though superior to that of the term or preterm newborns, did not differ significantly. The addition of adult serum had no effect on this function of the newborn's leucocytes.

These findings contrast with those of other investigators who, using various bacteria, found the ability of the leucocytes of newborn and particularly of preterm babies to phagocytize to be deficient and to improve considerably with the addition of adult serum (Forman and Stiehm, 1969; Miyamoto, 1965a, b; McCracken and Eichenwald, 1971). That serum opsonins are important for the phagocytosis of candida has been shown with mouse (Morelli and Rosenberg, 1971) and adult (Lehrer and Cline, 1969) leucocytes.

There is no doubt that the newborn's serum is deficient in opsonic activity (Dossett, 1972), and our findings regarding the $\mathrm{C} 3$ levels in the serum of the babies we examined confirm this finding. * However, Miller (1969) reported the newborn's serum opsonic deficiency to be apparent only when serum dilutions exceeded $10 \%$.

Our findings regarding efficient phagocytosis of candida by the newborn's leucocytes in their own serum could be explained in that the term or preterm babies' serum in the $25 \%$ dilution, which we used, provides adequate opsonins for a normal phagocytosis.

The ability of newborn leucocytes to kill the ingested candidae was found to be significantly lower compared with that of adult leucocytes.

Although serum factors seem to enhance the killing ability of newborn leucocytes towards various micro-organisms (Dossett et al., 1969; Forman and Stiehm, 1969; Park et al., 1970; McCracken and Eichenwald, 1971) exposure of our newborn leucocytes to adult serum did not improve their killing capacity. That the killing ability of candida is not influenced by serum factors has been shown by Morelli and Rosenberg (1971) with mice leucocytes and Lehrer and Cline (1969) with adult leucocytes. Our findings strongly suggest that the killing defect of newborn leucocytes towards $C$. albicans should not be sought in serum factors but in the leucocyte itself.

Of further interest is our finding that the leucocytes of the preterm babies are as efficient against $C$. albicans as those of the term babies. That preterm babies can be sensitized and produce antibodies as well as term babies has already been shown (Uhr, Dancis, and Newmann, 1960).

Finally, it should be noted that all these experiments were in vitro and that in vivo the deficiency of the newborn's leucocytes to handle candida infection may be further enhanced by the immature cell-mediated immunity of the newborn (Dossett, 1972; Matsaniotis, Economou-Mavrou, and Tsenghi, 1967).

REFERENCES

Dossett, J. H. (1972). Microbial defenses of the child and man. Pediatric Clinics of North America, 18, 355.

*Values in newborns of the present series are equivalent to $50 \%$ of adult values in the Greek population. 
Dossett, J. H., Williams, R. C., and Quie, P. G. (1969). Studies on interaction of bacteria, serum factors and polymorphonuclear leucocytes in mothers and newborns. Pediatrics, 44, 49.

Forman, M. L., and Stiehm, E. R. (1969). Impaired opsonic activity but normal phagocytosis in low-birth-weight infants. New England Fournal of Medicine, 281, 926.

Lehrer, R. I., and Cline, M. J. (1969). Interaction of Candida albicans with human leukocytes and serum. fournal of Bacteriology, 98, 996.

McCracken, G. H., and Eichenwald, H. F. (1971). Leukocyte function and the development of opsonic and complement activity in the neonate. American Fournal of Diseases of Children, $121,120$.

Matsaniotis, N., Economou-Mavrou, C., and Tsenghi, C. (1967). Low mitotic activity of peripheral lymphocytes during the first two years of life. Archives of Disease in Childhood, 42, 549.

Miller, M. E. (1969). Phagocytosis in the newborn infant: humoral and cellular factors. Fournal of Pediatrics, 74, 255.

Miyamoto, K. (1965a). Phagocytic activity of leukocytes in premature infants. Part I. Comparison of the phagocytic activity of leukocytes between premature infants and full-term infants. Hiroshima fournal of Medical Sciences, 14, 9.

Miyamoto, K. (1965b). Phagocytic activity of leukocytes in premature infants. Part II. Correlation of the plasma components and phagocytic activities of leukocytes in premature and full-term infants. Hiroshima fournal of Medical Sciences, 14, 19.

Morelli, R., and Rosenberg, L. T. (1971). The role of complement in the phagocytosis of Candida albicans by mouse peripheral blood leucocytes. Fournal of Immunology, 107, 476.

Park, B. H., Holmes, B., and Good, R. A. (1970). Metabolic activities in leukocytes of newborn infants. Fournal of Pediatrics, 76, 237.

Uhr, J. W., Dancis, J., and Neumann, C. G. (1960). Delayed-type hypersensitivity in premature neonatal humans. Nature, 187, 1130.

Correspondence to Dr. M. Xanthou, Department of Paediatrics, 'St. Sophie's' Children's Hospital, Athens 608, Greece. 